\title{
Anmerkungen zur Übersetzung italienischer Textstellen
}

In der vorliegenden Dissertation wird eine Übersetzung aus dem Italienischen ins Deutsche untersucht. Textstellen aus dem italienischen Originaltext werden daher im Folgenden häufig herangezogen und mit der entsprechenden deutschen Wiedergabe verglichen.

Um diesen Vergleich zwischen Ausgangs- und Zieltext auch für die nichtitalienischkundigen Leser fruchtbar zu machen, werden die Textstellen aus dem Novellino mit einer interlinearen Übersetzung ins Deutsche versehen. Dabei wird die Reihenfolge der italienischen Wörter beibehalten, und zwar auch da, wo dies gegen die Regeln der deutschen Wortstellung verstößt.

Damit die interlineare Version gut lesbar bleibt, habe ich i. d. R. auf morphologische Glossen verzichtet, zumal morphologische Aspekte in der vorliegenden Arbeit nicht in einer vergleichenden Perspektive behandelt werden sollen. Nur wenn unübersetzbare italienische Ausdrücke vorliegen sowie wenn die interlineare Version ohne morphologische Präzisierungen zweideutig gewesen wäre, habe ich auf morphologische Glossen nach den Richtlinien der Leipzig glossing rules (https://www.eva.mpg.de/lingua/resources/glossing-rules.php) zurückgegriffen. Das hier benutzte Verfahren möchte ich anhand einiger Beispiele näher erklären.

Wenn die deutschen Kasus die Funktion der italienischen Nominalphrasen passend wiedergeben konnten, habe ich diese benutzt, statt den Ausdruck im Nominativ anzugeben und sie mit einer morphologischen Glosse zu versehen. Im Beispiel stellt la battaglia das Objekt des Infinitivs rifiutare dar; der Ausdruck wurde durch das Akkusativobjekt den Kampf übersetzt.

Rifiutare la battaglia

[ablehnen den Kampf]

Wenn aus diesem Verfahren eine Zweideutigkeit resultiert, wurden die Nominalphrasen dagegen mit einer Glosse versehen: Die Weglassung des Subjekts egli in ui trouo vna donna bewirkt, dass eine Frau in der interlinearen Übersetzung als ein Nominativ interpretiert werden könnte; bei una donna handelt es sich jedoch um das Objekt des Verbs trouo. Deswegen wurde hier der Nominalphrase die Frau die Glosse [ACC] angehängt.

ui trouo vna donna

[dort fand eine Frau[ACC]

Im Italienischen - wie im Deutschen auch - können Präpositionen und Artikel verschmelzen. Wenn eine Verschmelzung im italienischen Ausgangstext vorliegt, die 
auch im Deutschen möglich ist, wird sie einfach durchgeführt, ansonsten werden Präposition und Artikel in der Übersetzung durch einen Bindestrich verbunden, um zu signalisieren, dass sie an der italienischen Textstelle verschmolzen sind:

Vn caualiere del Re

[Ein Ritter vom König]

Leggessi della bonta

[Liest-REFL von-der Güte]

Die beiden synthetischen Vergangenheitstempora des Italienischen, das imperfetto und das passato remoto, werden durch das Präteritum wiedergegeben; den analytischen Tempora entsprechen zusammengesetzte Verbformen: Das passato prossimo wird als Perfekt, das trapassato prossimo und remoto als Plusquamperfekt wiedergegeben. Da das Deutsche im Gegensatz zum Italienischen über kein synthetisches Futur verfügt, wird dieses glossiert. Dasselbe gilt für alle Verbformen, die keine Entsprechung im Deutschen haben.

$\begin{array}{llllll}\text { imp. } & \text { p. rem. } & \text { p. pross. } & \text { trap. pross./rem. } & \text { fut. } & \text { ger. } \\ \text { andavo } & \text { andai } & \text { sono andato } & \text { ero / fui andato } & \text { andrò } & \text { andando } \\ \text { ich ging } & \text { ging } & \text { bin gegangen } & \text { war gegangen } & \text { gehe-FUT } & \text { gehen-CVB }\end{array}$

Da das Subjekt im Italienischen weggelassen werden kann, wird in den Textauszügen aus dem Novellino das Zeichen $\emptyset$ benutzt, um die wahrscheinliche Stellung des fehlenden Subjekts zu signalisieren. Da die Frage der Identifizierung des Subjekts bei der Besprechung der Wiedergabe des gerundio in Kap. 4 relevant wird, wird dort nicht nur die Stellung des fehlenden Subjekts des gerundio hervorgehoben, sondern es wird auch das Bezugswort des gerundio im übergeordneten Satz indexiert:

Il padre vedendo $\emptyset_{\mathrm{i}}$ che catuno il volea. Mando per vn fine Orafo (LN, 72, p. 77)

Sofern nicht anders angegeben, stammen alle Übersetzungen der Zitate aus der Forschungsliteratur in italienischer Sprache von der Verfasserin. 